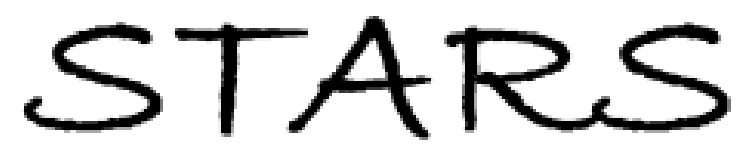

University of Central Florida

STARS

$1-1-2014$

\title{
Analysis of simulation methodology for calculation of the heat of transport for vacancy thermodiffusion
}

William C. Tucker

University of Central Florida

Patrick K. Schelling

University of Central Florida

Find similar works at: https://stars.library.ucf.edu/facultybib2010 University of Central Florida Libraries http://library.ucf.edu

This Article is brought to you for free and open access by the Faculty Bibliography at STARS. It has been accepted for inclusion in Faculty Bibliography 2010 s by an authorized administrator of STARS. For more information, please contact STARS@ucf.edu.

\section{Recommended Citation}

Tucker, William C. and Schelling, Patrick K., "Analysis of simulation methodology for calculation of the heat of transport for vacancy thermodiffusion" (2014). Faculty Bibliography 2010s. 6197.

https://stars.library.ucf.edu/facultybib2010/6197

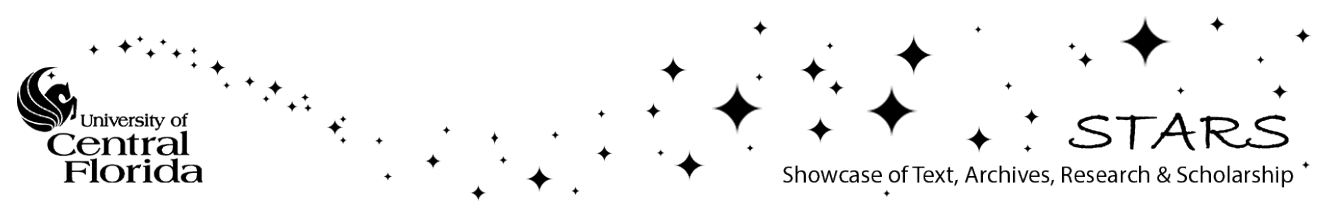




\section{Analysis of simulation methodology for calculation of the heat of transport for vacancy thermodiffusion}

Cite as: J. Appl. Phys. 116, 023504 (2014); https://doi.org/10.1063/1.4887121

Submitted: 19 March 2014 . Accepted: 24 June 2014 . Published Online: 09 July 2014

William C. Tucker, and Patrick K. Schelling

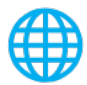

\section{ARTICLES YOU MAY BE INTERESTED IN}

Molecular-dynamics calculation of the vacancy heat of transport

Journal of Applied Physics 116, 023506 (2014); https://doi.org/10.1063/1.4886577

Atomic-scale simulation of the thermodiffusion of hydrogen in palladium

Journal of Applied Physics 114, 063509 (2013); https://doi.org/10.1063/1.4816961

Computational methodology for analysis of the Soret effect in crystals: Application to hydrogen in palladium

Journal of Applied Physics 112, 083516 (2012); https://doi.org/10.1063/1.4758462

\section{Applied Physics Reviews} Now accepting original research

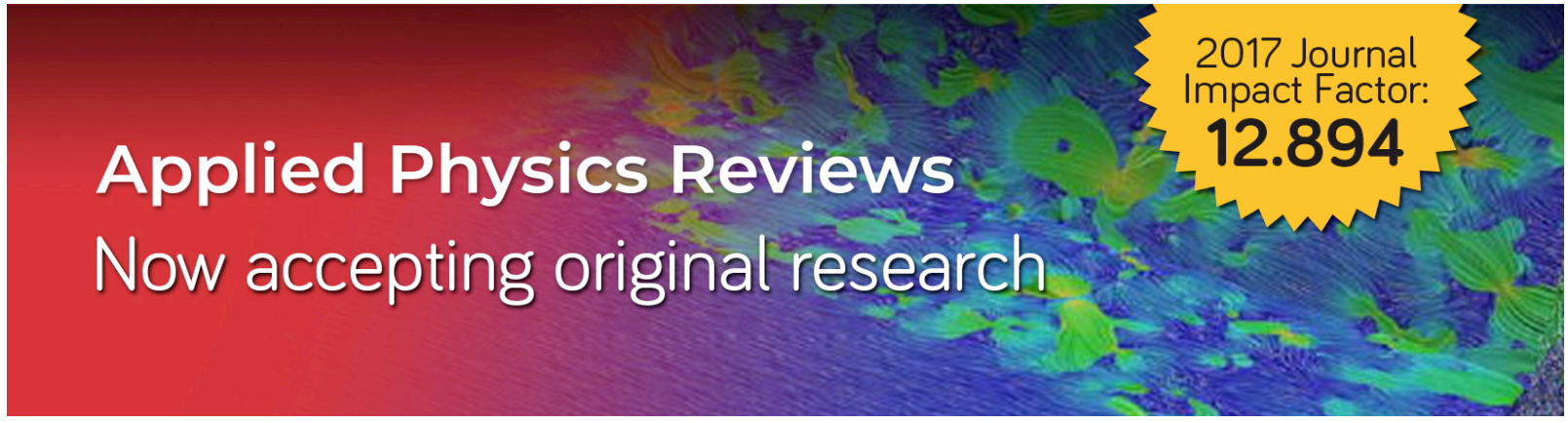




\title{
Analysis of simulation methodology for calculation of the heat of transport for vacancy thermodiffusion
}

\author{
William C. Tucker and Patrick K. Schellinga) \\ Advanced Material Processing and Analysis Center and Department of Physics, University of Central Florida, \\ 4000 Central Florida Blvd., Orlando, Florida 32816, USA
}

(Received 19 March 2014; accepted 24 June 2014; published online 9 July 2014)

\begin{abstract}
Computation of the heat of transport $Q_{a}^{*}$ in monatomic crystalline solids is investigated using the methodology first developed by Gillan [J. Phys. C: Solid State Phys. 11, 4469 (1978)] and further developed by Grout and coworkers [Philos. Mag. Lett. 74, 217 (1996)], referred to as the Grout-Gillan method. In the case of pair potentials, the hopping of a vacancy results in a heat wave that persists for up to $10 \mathrm{ps,} \mathrm{consistent} \mathrm{with} \mathrm{previous} \mathrm{studies.} \mathrm{This} \mathrm{leads} \mathrm{to} \mathrm{generally}$ positive values for $Q_{a}^{*}$ which can be quite large and are strongly dependent on the specific details of the pair potential. By contrast, when the interactions are described using the embedded atom model, there is no evidence of a heat wave, and $Q_{a}^{*}$ is found to be negative. This demonstrates that the dynamics of vacancy hopping depends strongly on the details of the empirical potential. However, the results obtained here are in strong disagreement with experiment. Arguments are presented which demonstrate that there is a fundamental error made in the Grout-Gillan method due to the fact that the ensemble of states only includes successful atom hops and hence does not represent an equilibrium ensemble. This places the interpretation of the quantity computed in the Grout-Gillan method as the heat of transport in doubt. It is demonstrated that trajectories which do not yield hopping events are nevertheless relevant to computation of the heat of transport $Q_{a}^{*}$. C 2014 AIP Publishing LLC. [http://dx.doi.org/10.1063/1.4887121]
\end{abstract}

\section{INTRODUCTION}

The coupled transport of heat and matter is generally referred to as thermodiffusion or the Ludwig-Soret effect. ${ }^{1}$ In a single-component material with an applied temperature gradient, mass transport occurs via the drift and diffusion of vacancies. The tendency of vacancies to drift in the presence of a temperature gradient is characterized by the reduced heat of transport of atoms, $Q_{a}^{* \prime}$, or alternately by the reduced heat of transport of vacancies, $Q_{v}^{* \prime}$. While this phenomenon is of considerable practical importance and has been the focus of many decades of investigation, there has been little progress made in developing a theoretical description from the atomic scale. The reason for this slow progress is that the characteristic hopping time for vacancies, $\sim 1$ ns or more, is too long for "brute force" simulation. This points to a need for methodological development to enable atomic-scale simulation to elucidate thermodiffusion.

To address the problem of vacancy-meditated thermodiffusion, Gillan ${ }^{2-4}$ developed an approach that was later extended and applied to finite temperatures for model fcc systems in Refs. 5-9. Hereafter, we refer to this approach as the Grout-Gillan method. The basic idea is to generate a very large ensemble of successful atomic hops. First, the system is equilibrated with the hopping atom constrained at the transition state. Next, the hopping atom is released, and the heat flux $\vec{J}_{q}$ is computed while the atom relaxes to one of the unoccupied neighboring fcc lattice sites. From a large

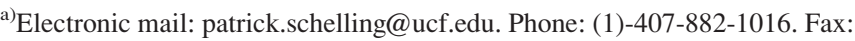
(1)-407-823-5112
}

ensemble of successful hops, the quantity $q(t)$ is computed from

$$
q(t)=\left\langle\left\langle\hat{e}_{0} \cdot \vec{J}_{q}(t)\right\rangle\right\rangle
$$

where $\hat{e}_{0}$ is a unit vector along the direction of the atom hop. The angle brackets indicate an average over an ensemble of successful hops. To obtain the heat of transport $Q_{a}^{*}$ for atoms, the function $q(t)$ is integrated

$$
Q_{a}^{*}=\frac{1}{s} \int_{-\tau}^{\tau} q(t) d t,
$$

where the integration limit $\tau$ should be large enough for the fluctuation to dissipate. Here, the quantity $s$ is the distance of the atomic hop. The association of the time-integrated heat flux with $Q_{a}^{*}$ is based on the phenomenological relation $\vec{J}_{q}=Q_{a}^{*} \vec{J}_{a}$, where $\vec{J}_{a}$ is the atom flux in a lattice-fixed reference frame. In Ref. 1, this relation is shown to apply when there is no temperature gradient, and the flux $\vec{J}_{a}$ occurs due to a concentration gradient or externally-applied force.

One characteristic feature of this approach observed in Refs. 5-9 is the presence of a large heat flux that persists $\sim 10$ ps both before and after the atom hop. It was found that this heat flux occurs in the same direction as that of the atom hop, yielding positive and often very large values for $Q_{a}^{*}$. The presence of a large heat flux was further elaborated in Ref. 9, where it was demonstrated that the calculation of $Q_{a}^{*}$ can exhibit large finite-size effects, with the computed values of $Q_{a}^{*}$ increasing significantly with system size especially at low temperatures. These results suggest that for vacancymediated thermodiffusion, $Q_{a}^{*}$ should always be positive, with a magnitude that depends very strongly on temperature. 
In this paper, it is demonstrated that $Q_{a}^{*}$ computed using the Grout-Gillan method is strongly dependent on the details of the interatomic potential. Specifically, it is shown that pair potentials similar to those used in Refs. 5-9 lead to positive values for the reduced heat of transport $Q_{a}^{* \prime}=Q_{a}^{*}-h_{a}+h_{v}$, but with magnitudes that can vary significantly depending on details of the particular potential. In addition, results for an embedded-atom method (EAM) potential for gold are reported, which demonstrate that negative values are possible for $Q_{a}^{* \prime}$. In short, it is shown that the trends reported in Refs. 5-9 are specific to pair potentials, while EAM potentials thought to be more realistic for the modeling of vacancies, result in very different qualitative behavior. However, using the Grout-Gillan method with EAM potentials is still found to result in predictions in significant disagreement with experiment.

We explore features of the ensemble of states obtained by the Grout-Gillan method. Because the method is based on diffusion in the absence of a temperature gradient, it is implicit that the results should be obtained from an equilibrium ensemble. Here, two important properties of the equilibrium statistical ensemble are identified. First, atomic velocities should be uncorrelated with atomic coordinates at each instant of time within the sampled ensemble of trajectories. Second, the velocity components of all the atoms should be uncorrelated within the ensemble. It is demonstrated that these requirements are not met by the Grout-Gillan ensemble, which brings into question the interpretation of Eq. (2) as the heat of transport $Q_{a}^{*}$. We suggest that some of the unusual features seen with the Grout-Gillan method, including the apparent failures in obtaining agreement with experiment, are directly the result of sampling an incomplete ensemble of states. The proper ensemble of states should also include trajectories that might result in large fluctuations and lattice heat conduction, but failed atomic hops. Hence, we argue that $Q_{a}^{*}$ computed from Eqs. (1) and (2) should not be interpreted as the heat of transport when the states used to compute $q(t)$ are taken from the ensemble generated by the Grout-Gillan method.

\section{SIMULATION APPROACH}

We apply the Gillan-Grout method as described above in Eqs. (1) and (2). The general approach is fully described in Refs. 2-9. The method applied here for constraining the atom at the transition state is somewhat different from Refs. 5-9, but we believe that any differences are inconsequential. The approach was described fully in Ref. 10, but for completeness we describe the essential features here. The hopping atom starts at the transition state exactly between two unoccupied fcc lattice sites connected along a [110] direction. At each step, the force $\vec{F}_{1}$ on the hopping atom $(i=1)$ due to the other atoms is computed. The constraint force required to hold the hopping atom at the transition state is given by

$$
\vec{F}_{c}=-\left(\vec{F}_{1} \cdot \hat{e}_{0}\right) \hat{e}_{0}
$$

where $\hat{e}_{0}$ is a unit vector along the $z$-direction of the simulation cell, which also corresponds to the [110] crystallographic direction of the atom hop. The force on the hopping atom is then found by adding the constraint force

$$
\vec{F}_{1}^{\prime}=\vec{F}_{1}+\vec{F}_{c}
$$

As the hopping atom follows the dynamics prescribed by the force $\vec{F}_{1}^{\prime}$, there is no component of the force acting along the direction of the hop, thereby constraining the atom at the transition state. However, the other atoms in the cell should also be constrained. For these atoms $(i \neq 1)$, we also applied a constraint force, and the dynamics were determined from the forces $\vec{F}_{i}^{\prime}$ given by

$$
\vec{F}_{i}^{\prime}=\vec{F}_{i}-\frac{\vec{F}_{c}}{N-1}
$$

where $\vec{F}_{i}$ is the force on atom $i$ due to all of the other $N-1$ atoms in the simulation cell. It is easy to see that the net force on the system is zero, $\sum_{i=1}^{N} \vec{F}_{i}^{\prime}=0$. After this initial simulation period, the net momentum is zero and the hopping atom is constrained at the transition state. Because only one degree of freedom is held fixed, the other degrees of freedom have sufficient freedom to relax. The hopping atom $(i=1)$ moves freely in a (110) plane.

After equilibration at the transition state, the constraint force is removed and the hopping atom is given a velocity along the [110] crystallographic direction drawn from a Boltzmann distribution. In the following, we associate the z-axis of a Cartesian system with the [110] direction. Then the z-component, $v_{z}$, of the velocity of the hopping atom is drawn randomly with probability of a value between $v_{z}$ and $v_{z}+d v_{z}$ given by

$$
P\left(v_{z}\right) d v_{z}=\left(\frac{m}{2 \pi k_{B} T}\right)^{1 / 2} \exp \left(-\frac{m v_{z}^{2}}{2 k_{B} T}\right) d v_{z} .
$$

The sign of $v_{z}$ can be either positive or negative, and hence the direction of the atom hop is not predetermined. After removing the constraint force $\vec{F}_{c}$, the subsequent dynamics results in the hopping atom relaxing to one of the neighboring fcc lattice sites. However, at each MD step, $\vec{F}_{c}$ is still computed according to Eq. (3), and the dynamics of the other $N-1$ atoms are determined by the forces $\vec{F}_{i}^{\prime}$ obtained from Eq. (5). Consequently, the component of the net momentum along the direction of the atom hop for the $N-1$ lattice atoms is zero and does not change with time, consistent with a lattice-fixed reference frame. Because the hopping atom carries a net momentum during the hopping process, the center-of-mass of the entire system including the hopping atom is not fixed. This is in contrast to the previous applications of the Gillan-Grout method, where the simulations were conducted in a center-of-mass reference frame. ${ }^{5-9}$

It is still necessary to determine whether the configuration prepared in this way results in a successful hop. To establish whether or not a successful hop has occurred, we also reverse all of the atomic velocities at the transition state and simulate the resulting trajectory. This is equivalent to running the calculation backwards in time. In this way, a complete trajectory from the initial to the final state is determined. Successful hops are included in the ensemble 
when the magnitude of the displacement of the hopping atom is found to be greater than one-half of the distance separating the two vacant fcc lattice sites.

After releasing the hopping atom from the transition state, the energy flux is computed as a function of time. We report simulation results for pair potentials and also a potential for $\mathrm{Au}$ based on the EAM formalism. These potentials require different expressions for the energy flux $\vec{J}_{q}$. In the case of a pair potential, the total energy of the system is given by

$$
E_{t o t}=\frac{1}{2} \sum_{i} m \vec{v}_{i} \cdot \vec{v}_{i}+\sum_{\langle i j\rangle} \phi\left(r_{i j}\right)
$$

where $m$ is the atomic mass, $\vec{v}_{i}$ is the velocity vector of atom $i, \phi(r)$ is the pair potential energy, $r_{i j}$ is the separation between atoms $i$ and $j$, and the summation in the second term is over all pairs $\langle i j\rangle$. The energy flux $\vec{J}_{q}$ is computed using

$$
\vec{J}_{q}=\sum_{i} \epsilon_{i} \vec{v}_{i}-\frac{1}{2} \sum_{\langle i j\rangle} \vec{r}_{i j}\left[\frac{1}{r} \frac{d \phi}{d r}\right]_{r=r_{i j}}\left[\vec{r}_{i j} \cdot\left(\vec{v}_{i}+\vec{v}_{j}\right)\right],
$$

where $\vec{r}_{i j}=\vec{r}_{i}-\vec{r}_{j}$, and the local energy $\epsilon_{i}$ is given by

$$
\epsilon_{i}=\frac{1}{2} m \vec{v}_{i} \cdot \vec{v}_{i}+\frac{1}{2} \sum_{j \neq i} \phi\left(r_{i j}\right)
$$

The choice that the potential energy is equally shared between the interacting atoms $i$ and $j$ is quite standard, and given this assumption the energy flux expression can be derived using the formalism in Ref. 11.

For simulations using the EAM potential, the total energy is given by

$$
E_{t o t}=\frac{1}{2} \sum_{i} m \vec{v}_{i} \cdot \vec{v}_{i}+\sum_{i} F\left(\bar{\rho}_{i}\right)+\sum_{\langle i j\rangle} \phi\left(r_{i j}\right) .
$$

Here, the function $F(\rho)$ is the embedding energy which depends on the charge density $\rho$ due to the neighboring atoms. The local charge $\bar{\rho}_{i}$ at site $i$ due to the neighboring atoms $j$ given by

$$
\bar{\rho}_{i}=\sum_{j \neq i} \rho\left(r_{i j}\right)
$$

where $\rho(r)$ is an empirical function for the charge density due to an atom at radial distance $r$. Again using the formalism in Ref. 11, the energy flux is given by

$$
\begin{aligned}
\vec{J}_{q}= & \sum_{i} \epsilon_{i} \vec{v}_{i}-\frac{1}{2} \sum_{\langle i j\rangle} \vec{r}_{i j}\left[\frac{1}{r} \frac{d \phi}{d r}\right]_{r=r_{i j}}\left[\vec{r}_{i j} \cdot\left(\vec{v}_{i}+\vec{v}_{j}\right)\right] \\
& -\sum_{i} \vec{r}_{i j}\left[\frac{d F}{d \rho}\right]_{\rho=\bar{\rho}_{i}}\left[\frac{1}{r} \frac{d \rho}{d r}\right]_{r=r_{i j}}\left(\vec{r}_{i j} \cdot \vec{v}_{j}\right) .
\end{aligned}
$$

The local energy of site $i$ is given by

$$
\epsilon_{i}=\frac{1}{2} m \vec{v}_{i} \cdot \vec{v}_{i}+F\left(\bar{\rho}_{i}\right)+\frac{1}{2} \sum_{j \neq i} \phi\left(r_{i j}\right) .
$$

In deriving these expressions for the EAM potentials, the embedding energy $F\left(\bar{\rho}_{i}\right)$ is entirely assigned to site $i$, whereas pairwise potential energy $\phi\left(r_{i j}\right)$ is evenly divided between the two interacting sites. As with a simple pair potential, these equations are not unique. However, given the assignment of the local energy in Eq. (13), the expression for $\vec{J}_{q}$ can be determined in a straightforward way from the formalism in Ref. 11.

Finally, we identify individual convective and virial contributions. In both the pair and EAM potentials, the convective term in the energy flux $\vec{J}_{q}^{(\text {conv })}$ is given by

$$
\vec{J}_{q}^{(\text {conv })}=\sum_{i} \epsilon_{i} \vec{v}_{i}
$$

and the virial energy flux $\vec{J}_{q}^{(v i r)}$ is given by the remaining terms, so that

$$
\vec{J}_{q}^{(v i r)}=\vec{J}_{q}-\vec{J}_{q}^{(\text {conv })}
$$

where $\vec{J}_{q}$ is the total energy flux given by Eq. (8) for pair potentials and Eq. (12) for EAM potentials.

The method used to determine the partial energies, $u_{a}$ and $u_{v}$, and enthalpies, $h_{a}$ and $h_{v}$, is described next. It is necessary to compute the energy and pressure of a perfect crystal with $N_{0}$ lattice sites and $N=N_{0}$ atoms, and also a defective crystal with $N_{0}$ lattice sites occupied by $N=N_{0}-1$ atoms with one vacant lattice site. To obtain the correct quantities for computation of the reduced heat of transport $Q_{a}^{* \prime}=Q_{a}^{*}-h_{a}+h_{v}$, the calculations for the perfect crystal and defective system are performed at the same volume. First, the partial energies $u_{N_{0}}$ and $u_{N_{0}-1}$ of the perfect and defective systems are determined from

$$
\begin{gathered}
u_{N_{0}}=\frac{U_{N_{0}}}{N_{0}}, \\
u_{N_{0}-1}=\frac{U_{N_{0}-1}}{N_{0}-1},
\end{gathered}
$$

where $U_{N_{0}}$ is the total energy of the perfect crystal with $N=N_{0}$ atoms, and $U_{N_{0}-1}$ is the total energy of the system with $N=N_{0}-1$ atoms and one vacancy. The partial energy $u_{a}$ for the atoms is found from

$$
u_{a}=u_{N_{0}} .
$$

The partial energy of vacancy $u_{v}$ is found by removing an atom from a perfect crystal and placing it into a reference state of perfect crystal. Hence

$$
u_{v}=\left(u_{N_{0}-1}-u_{N_{0}}\right)\left(N_{0}-1\right) .
$$

The total enthalpy in the system with $N=N_{0}$ atoms is given by

$$
H_{N_{0}}=U_{N_{0}}+p_{N_{0}} \Omega
$$

Similarly, the total enthalpy in the system with $N=N_{0}-1$ atoms and a vacancy is given by

$$
H_{N_{0}-1}=U_{N_{0}-1}+p_{N_{0}-1} \Omega .
$$


In these expressions, $\Omega$ is the system volume, which is the same for the perfect and defective systems. Since the system volume $\Omega$ is the same in the perfect and defective systems, the pressures $p_{N_{0}}$ and $p_{N_{0}-1}$ are not equal. The partial enthalpies $h_{N_{0}}$ and $h_{N_{0}-1}$ for the perfect and defective systems are found from

$$
\begin{gathered}
h_{N_{0}}=\frac{H_{N_{0}}}{N_{0}}, \\
h_{N_{0}-1}=\frac{H_{N_{0}-1}}{N_{0}-1} .
\end{gathered}
$$

Then the partial enthalpy of an atom is

$$
h_{a}=h_{N_{0}} .
$$

Similarly, the partial enthalpy of a vacancy is,

$$
h_{v}=\left(h_{N_{0}-1}-h_{N_{0}}\right)\left(N_{0}-1\right) \text {. }
$$

The relevant quantity for the transport parameters in the lattice-fixed frame is the difference $h_{a}-h_{v}$. Specifically, the reduced heat of transport is given by $Q_{a}^{* \prime}=Q_{a}^{*}-h_{a}+h_{v}$. This is due to the fact that, in a lattice-fixed reference frame, there is a net atom and vacancy flux. In a center of mass frame, relevant for the simulations reported in Refs. 5-9, there is no net atom flux and only $h_{v}$ is relevant for computing the reduced heat of transport. Hence, for simulations in a center-of-mass reference frame, the reduced heat of transport should be determined using $Q_{a}^{* \prime}=Q_{a}^{*}+h_{v}$. In computing the reduced heat of transport, the factor $h_{v}$ was not included in Refs. 5-9. We believe this was an error in those works. For the remainder of the paper, in comparing the present results for $Q_{a}^{* \prime}$ using the Cherns potential ${ }^{12}$ with Ref. 9, we include the missing factor $h_{v}$.

\section{RESULTS}

We first present simulation results using the Cherns potential for $\mathrm{Au}^{12}$ studied in Refs. 8 and 9. The system included $N_{0}=288$ lattice sites and $N=N_{0}-1=287$ atoms. The $\mathrm{x}^{-}, \mathrm{y}-$, and z-directions of a Cartesian system were chosen coincident with the [110], [001], and [110] crystallographic directions. The system dimensions were $3 \sqrt{2} a$ in the $x$ - and $z$-directions, and $4 a$ in the $y$-direction, with $a=4.08 \AA$. The atom hop was along the $z$-direction. The system was first equilibrated at the transition state for $10^{4} \mathrm{MD}$ steps of length $\Delta t=1.17$ fs. For each state equilibrated in this way, there were 10 attempts at a hop. Approximately $55 \%$ of the attempts resulted in a successful hop, hence about $1.43 \times 10^{4}$ trajectories were included in the ensemble average. The average temperature was taken to be $T=300 \mathrm{~K}$. The simulation was run for $19.49 \mathrm{ps}$ forward and backward in time from the transition state to determine the energy flux $\vec{J}_{q}$.

In Fig. 1, we show $q(t)$ averaged over the ensemble of successful hops, and also separately the virial and convective parts in Fig. 2. Integration according to Eq. (2) using limit $\tau=12.0$ ps yields $Q_{a}^{*}=11.81 \pm 0.06 \mathrm{eV}$. Further insight can be obtained from analysis of the separate contributions $\vec{J}_{q}^{(\text {conv })}$ and $\vec{J}_{q}^{(v i r)}$ to the integral in Eq. (2), so that $Q_{a}^{*}$

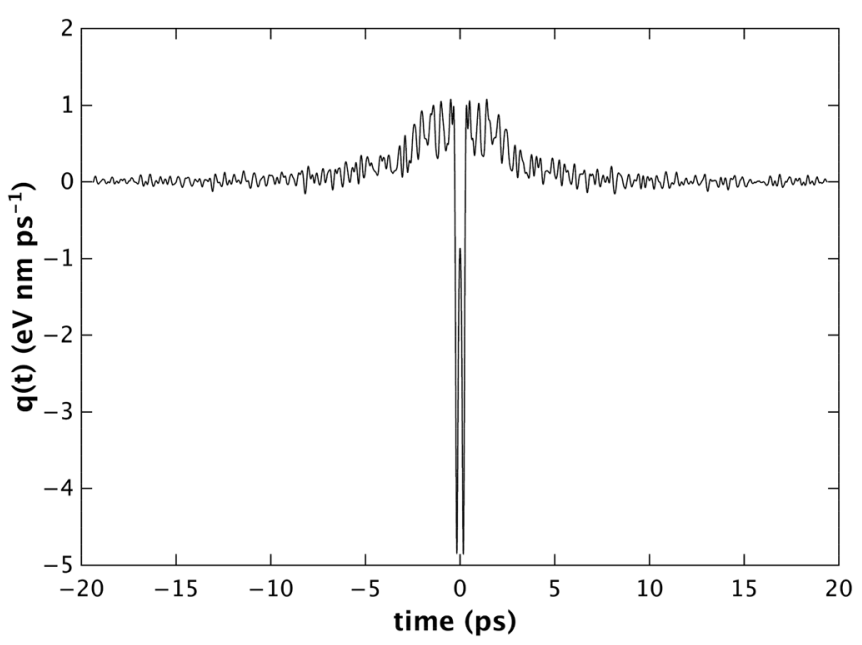

FIG. 1. The function $q(t)$ from Eq. (1) plotted as a function of time averaged over an ensemble of hops obtained with $T=300 \mathrm{~K}$ for $N_{0}=288 \mathrm{fcc}$ lattice sites and $N=N_{0}-1$ atoms. The results here are for the Cherns potential parameterized for $\mathrm{Au} .^{12}$

$=Q_{a}^{*(\text { conv })}+Q_{a}^{*(v i r)}$. From the individual contributions, it is found that the virial contribution is positive, $Q_{a}^{*(v i r)}$ $=13.37 \pm 0.04 \mathrm{eV}$, and the convective part is negative, $Q_{a}^{*(\text { conv })}=-1.57 \pm 0.02 \mathrm{eV}$. The error bars here and elsewhere are determined from sampling subsets of the total ensemble. It is evident that the convective part is only important during the actual hop, whereas the virial part persists for $10 \mathrm{ps}$ before and after the hopping event. These results are generally consistent with the results reported in Refs. 5-9. The hop itself occurs over a time interval less than $\sim 1$ ps. During the hop, the direction of the energy flux is opposite the direction of the hop, resulting in negative values for $q(t)$. After the hop occurs, there remains a positive energy flux due to the virial contribution, indicating that this is conductive in nature. By time-reversal symmetry, verified by the results in Figs. 1 and 2, $q(t)=q(-t)$, and the energy flux before the hop is also in the same direction as the atom hop.

The reduced heat of transport given by $Q_{a}^{* \prime}=$ $Q_{a}^{*}-h_{a}+h_{v}$ is an even larger positive quantity. The value

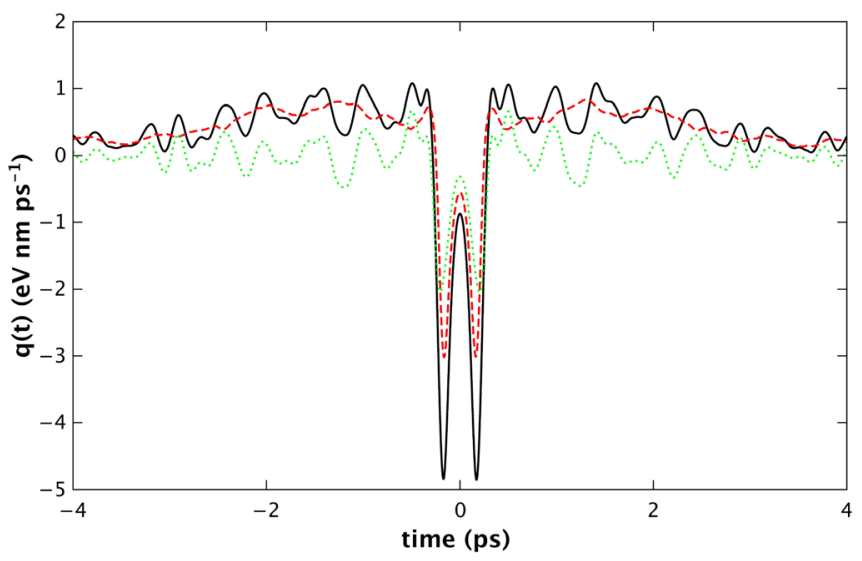

FIG. 2. Contributions to the function $q(t)$ (solid black line) from the virial flux $\vec{J}_{q}^{(v i r)}$ (red dashed line) and convective energy flux $\vec{J}_{q}^{(\text {conv })}$ (green dotted line). The simulation conditions correspond to those in Fig. 1. 
TABLE I. Computed transport parameters for the Cherns, Morse, and EAM potentials. The Morse potentials include different values of the parameter $\alpha r_{0}$. Results are given for different temperatures, lattice parameters $a$, and system sizes $N_{0}$. Both the heat of transport $Q_{a}^{*}$ and reduced heat of transport $Q_{a}^{* \prime}=$ $Q_{a}^{*}-h_{a}+h_{v}$ are reported.

\begin{tabular}{|c|c|c|c|c|c|c|}
\hline Potential & $\mathrm{T}(\mathrm{K})$ & $a(\AA)$ & $N_{0}$ & $h_{a}-h_{v}(\mathrm{eV})$ & $Q_{a}^{*}(\mathrm{eV})$ & $Q_{a}^{* \prime}(\mathrm{eV})$ \\
\hline Cherns $^{12}$ & 300 & 4.080 & 288 & -7.02 & $11.81 \pm 0.06$ & $18.83 \pm 0.06$ \\
\hline Morse $\alpha r_{0}=4.55$ & 1016 & 4.082 & 1400 & -7.83 & $-0.59 \pm 1.58$ & $7.24 \pm 1.58$ \\
\hline Morse $\alpha r_{0}=6.00$ & 1016 & 4.249 & 1400 & -7.83 & $17.27 \pm 0.91$ & $25.09 \pm 0.91$ \\
\hline Morse $\alpha r_{0}=8.00$ & 1016 & 4.301 & 1400 & -7.56 & $37.18 \pm 0.96$ & $44.74 \pm 0.96$ \\
\hline Morse $\alpha r_{0}=4.55$ & 1354 & 4.082 & 1400 & -8.14 & $-1.48 \pm 1.28$ & $6.65 \pm 1.28$ \\
\hline Morse $\alpha r_{0}=6.00$ & 1354 & 4.249 & 1400 & -6.58 & $11.04 \pm 1.12$ & $17.62 \pm 1.12$ \\
\hline Morse $\alpha r_{0}=8.00$ & 1354 & 4.301 & 1400 & -5.85 & $28.84 \pm 0.90$ & $34.69 \pm 0.90$ \\
\hline $\mathrm{EAM}^{14}$ & 700 & 4.070 & 1400 & -2.20 & $-6.21 \pm 0.27$ & $-4.00 \pm 0.27$ \\
\hline $\mathrm{EAM}^{14}$ & 700 & 4.090 & 1400 & -2.93 & $-6.56 \pm 0.16$ & $-3.62 \pm 0.16$ \\
\hline
\end{tabular}

$h_{a}-h_{v}=-7.02 \mathrm{eV}$ was obtained from runs of $3 \times 10^{5} \mathrm{MD}$ steps, with the first $1 \times 10^{5}$ steps used for equilibration and not included in the averages. The reduced heat of transport is then $Q_{a}^{* \prime}=18.83 \pm 0.06 \mathrm{eV}$. These values are summarized in Table I. These results highlight the extremely large values that are characteristic of the Grout-Gillan method primarily due to the very large positive virial contribution. In Ref. 9, the values $Q_{a}^{*}$ were computed in a center-of-mass reference frame for various temperatures and system sizes. The result $Q_{a}^{*} \approx 9.0 \mathrm{eV}$ from Ref. 9 obtained from a system with $N_{0}=256$ lattice sites at temperature $T=450 \mathrm{~K}$ is comparable to the result presented here. For simulations run in the center-of-mass reference frame, $Q_{a}^{* \prime}=Q_{a}^{*}+h_{v}$, which results in the value $Q_{a}^{* \prime} \approx 12 \mathrm{eV}$. At higher temperatures in Ref. 9, the value of $Q_{a}^{*}$ strongly decreases. For example, at $T=1100 \mathrm{~K}$, $Q_{a}^{*} \approx 3 \mathrm{eV}$ was computed, corresponding to a reduced heat of transport $Q_{a}^{* \prime} \approx 6 \mathrm{eV}$.

Simulations using different Morse potentials, each with the functional form

$$
V(r)=\epsilon\left[e^{-2 \alpha\left(r-r_{0}\right)}-2 e^{\alpha\left(r-r_{0}\right)}\right]
$$

were also performed. Three different values for $\alpha r_{0}$ were chosen, including $\alpha r_{0}=4.55, \alpha r_{0}=6.0$, and $\alpha r_{0}=8.0$. The value $\alpha r_{0}=4.55$ is the same value as reported in the Cherns potential for $\mathrm{Au}$. The range of the potential was limited by a cutoff $r_{\text {cut }}=3 r_{0}$, using a standard smoothing procedure to eliminate discontinuities in the potential and forces at

TABLE II. Potential parameters and resulting material properties for simulations using the Morse potentials. The cohesive energy is computed at $T=0 \mathrm{~K}$ using the zero-pressure lattice parameters $a$ obtained for $T=1016 \mathrm{~K}$. The bulk modulus $B_{0}$ and activation energies $\Delta U_{a}$ and $\Delta A_{a}$ were computed at $T=1016 \mathrm{~K}$.

\begin{tabular}{lccc}
\hline \hline Parameter & $\alpha r_{0}=4.55$ & $\alpha r_{0}=6.0$ & $\alpha r_{0}=8.0$ \\
\hline$\alpha\left(\AA^{-1}\right)$ & 1.4992 & 1.9769 & 2.6359 \\
$\epsilon(\mathrm{eV})$ & 0.5838 & 0.5838 & 0.5838 \\
$r_{0}(\AA)$ & 3.0350 & 3.0350 & 3.0350 \\
$B_{0}(\mathrm{GPa})$ & 180.0 & 229.9 & 355.0 \\
$a(\AA)$ & 4.08 & 4.25 & 4.31 \\
$E_{c o h}(\mathrm{eV})$ & 4.85 & 4.01 & 3.66 \\
$\Delta A_{a}(\mathrm{eV})$ & $1.762 \pm 0.001$ & $2.819 \pm 0.001$ & $4.319 \pm 0.001$ \\
$\Delta U_{a}(\mathrm{eV})$ & $1.84 \pm 0.01$ & $2.84 \pm 0.01$ & $4.23 \pm 0.01$ \\
\hline \hline
\end{tabular}

$r=r_{\text {cut }}$. The parameters $r_{0}=3.035 \AA$ and $\epsilon=0.5838 \mathrm{eV}$ were chosen. With this parameterization, the potential with $\alpha r_{0}=4.55$ corresponds fairly closely to $\mathrm{Au}$, resulting in a lattice parameter $a=4.08 \AA$ and bulk modulus $B_{0}=180 \mathrm{GPa}$ at $T=1016 \mathrm{~K}$. The potentials with $\alpha r_{0}=6.0$ and $\alpha r_{0}=8.0$ were not chosen to represent any real material, but rather to determine systematically how the computed values of $Q_{a}^{*}$ depend upon parameterization of the pair potential. In each instance, the atomic mass was taken to be that of Au. The particular values of the parameters used for the three Morse potentials are presented in Table II, along with computed values of the lattice parameter $a$, bulk modulus, and cohesive energy $E_{c o h}$. The cohesive energies were computed at $T=0 \mathrm{~K}$ for each of the lattice parameters in Table II. For the $\alpha r_{0}=4.55$ potential that corresponds closely to $\mathrm{Au}$, the cohesive energy computed using the the Morse potential is significantly larger than the experimental value $E_{c o h}=3.81 \mathrm{eV} .{ }^{13}$ The computed values of the activation energy $\Delta U_{\text {act }}$ and activation free energy $\Delta A_{a c t}$ are also reported in Table II. The activation energies were computed at $T=1016 \mathrm{~K}$ by computing the energy and potential of mean force as a function of reaction coordinate $z$ of an atom neighboring a vacancy.

In Fig. 3, the function $q(t)$ is shown for the potential $\alpha r_{0}=4.55$ at temperature $T=1016 \mathrm{~K}$. The results in Fig. 3

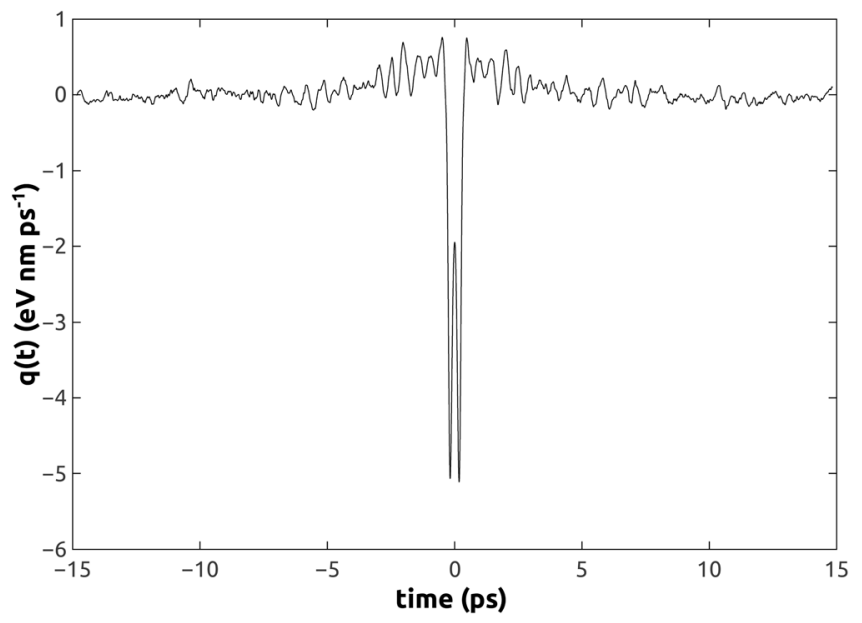

FIG. 3. The function $q(t)$ from Eq. (1) plotted as a function of time averaged over an ensemble of hops obtained with $T=1016 \mathrm{~K}$ for $N_{0}=1400 \mathrm{fcc}$ lattice sites and $N=N_{0}-1$ atoms. The results here are obtained using the Morse potential with parameterization $\alpha r_{0}=4.55$. 
and for each of the simulations using the Morse potentials were averaged over approximately $4 \times 10^{4}$ successful hops. The system orientation was the same as that used for the Cherns potential, but a total of $N_{0}=1400$ lattice sites were included, with system dimensions $5 \sqrt{2} a, 7 a$, and $5 \sqrt{2} a$ along the $x-, y-$ and $z$-directions, respectively. The system was first equilibrated at the transition state for $2.2 \times 10^{4} \mathrm{MD}$ steps of length $\Delta t=1.236 \mathrm{fs}$. For each state equilibrated in this way, there were 4 attempts at a hop, with approximately $82 \%$ of the attempts resulting in a successful hop. The simulation was run for $15 \mathrm{ps}$ forward and backward in time from the transition state to determine the energy flux $\vec{J}_{q}$ which was averaged over all successful hops. Values of $Q_{a}^{*}$ were obtained using integration limits $\tau=10 \mathrm{ps}$ in Eq. (2). Simulation results using the Morse potentials with different values of $\alpha r_{0}$ and temperatures $T=1016 \mathrm{~K}$ and $T=1354 \mathrm{~K}$ are reported in Table I. Qualitatively, the results are similar to those obtained using the Cherns potential. Specifically, the virial energy flux is dominant, and with the exception of $\alpha r_{0}=4.55$, this results in very large, positive values computed for $Q_{a}^{*}$. The computed values for $Q_{a}^{*}$ can be extremely large. For example, for $\alpha r_{0}=8.0$ at $T=1016 \mathrm{~K}, Q_{a}^{*}=$ $37.18 \pm 0.96 \mathrm{eV}$ was obtained. The dependence of $Q_{a}^{*}$ on the parameter $\alpha r_{0}$ is easily understood. Specifically, as $\alpha r_{0}$ increases, the activation free energy and bulk modulus both increase, as shown in Table II. The barrier height closely corresponds to the amount of energy transferred between the lattice and the hopping atom. As the barrier height increases, the magnitude of the virial heat conduction increases. The virial heat conduction also depends on speed of propagation of lattice waves, which depends on the elastic properties of the lattice. Hence, as $\alpha r_{0}$ increases and the bulk modulus increases, the heat wave in the lattice propagates more effectively and the computed value $Q_{a}^{*}$ increases. Together, these factors are responsible for the dramatic increases in $Q_{a}^{*}$ with the parameter $\alpha r_{0}$ shown in Table I. The computed reduced heats of transport $Q_{a}^{* \prime}$ reported in Table I are positive and quite large in each case.

The dependence on temperature in Table I using the Morse potentials can be easily understood. The dominant factor that controls the temperature dependence is that for higher temperatures, the heat wave, corresponding to lattice heat conduction, is scattered more effectively at higher temperatures. As a result, $Q_{a}^{*}$ tends to decrease strongly with temperature. This effect was also reported in Ref. 9 for simulations using the Cherns potential. We have not explored system-size dependence, as was done in Ref. 9. The systems simulated here were larger than those reported in Ref. 9, and hence we expect that system-size dependence may not be too dramatic. However, the effect of increasing system size should be to gradually increase the computed values $Q_{a}^{*}$, trending towards even larger positive values.

Next, we present results obtained from simulations at $T=700 \mathrm{~K}$ using the EAM potential for Au developed in Ref. 14. Higher temperatures were attempted, but it was found that at higher temperatures the atomic hop could cause local disordering of the lattice. The system size and orientation was identical to that described above for the Morse potential simulations. The system was first equilibrated at the

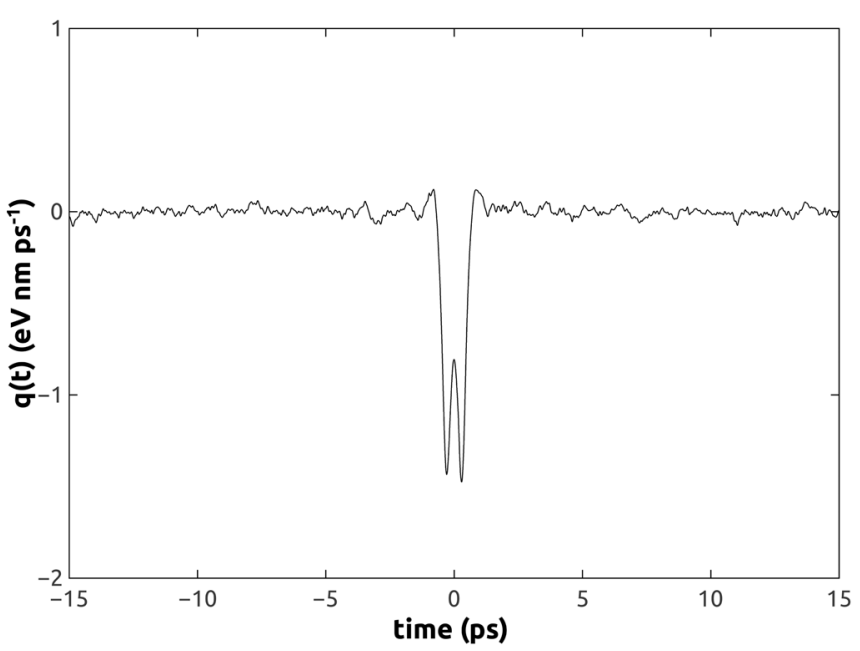

FIG. 4. The function $q(t)$ from Eq. (1) plotted as a function of time averaged over an ensemble of hops obtained with $T=700 \mathrm{~K}$ for $N_{0}=1400$ fcc lattice sites and $N=N_{0}-1$ atoms. The results here are obtained using the EAM potential for Au described in Ref. 14.

transition state for $1.9 \times 10^{4} \mathrm{MD}$ steps of length $\Delta t=1.0 \mathrm{fs}$. Again 4 attempts at a hop were attempted for each equilibrated transition state, with approximately $75 \%$ of the attempts resulting in a successful hop. Results were obtained for lattice parameter $a=4.07 \AA$, and also $a=4.09 \AA$ which corresponded closely to zero pressure at $T=700 \mathrm{~K}$. The simulation was run for $15 \mathrm{ps}$ forward and backward in time from the transition state. In Fig. 4, the function $q(t)$ is displayed. The results indicate that the virial heat flux does not evidently play a dominant role for simulation with the EAM potential. The computed values of $Q_{a}^{*}$ for two different lattice parameters at $T=700 \mathrm{~K}$ are shown in Table I. In contrast to the results obtained using pair potentials, the predicted $Q_{a}^{*}$ values are strongly negative. The values for $h_{a}-h_{v}$ were obtained from runs of $10^{6} \mathrm{MD}$ steps, with the first $10^{5}$ steps used for equilibration and not included in the averages. The computed values for $Q_{a}^{* \prime}$, shown in Table I, are also negative.

Additional features of the simulations using the EAM potential are revealed by considering specific contributions to the heat flux $\vec{J}_{q}$. The convective part of the heat transport can be separated into kinetic and potential energy contributions. The virial contribution to the heat flux can also be separated into contributions due to the pair potential and the embedding energy. In Fig. 5, the individual contributions to the integral in Eq. (2) are shown as a function of the integration limit $\tau$. The kinetic energy contribution is positive, as expected, and slightly less than the computed activation energy $\Delta U_{a c t}=0.60 \mathrm{eV}$. The energy flux due to convection of the potential energy is further divided into contributions due to the pair potential and embedding energy. Both convective potential energy contributions make negative contributions to the $Q_{a}^{*}$, with the embedding energy playing the largest role. Similarly, the contributions from the pair potential and embedding energy to the virial energy flux are both depicted in Fig. 5. Unlike the results obtained using pair potentials, the virial contributions are not dominant. In fact, both virial contributions are negative, and hence there is no evidence of a large heat wave propagating in the same 


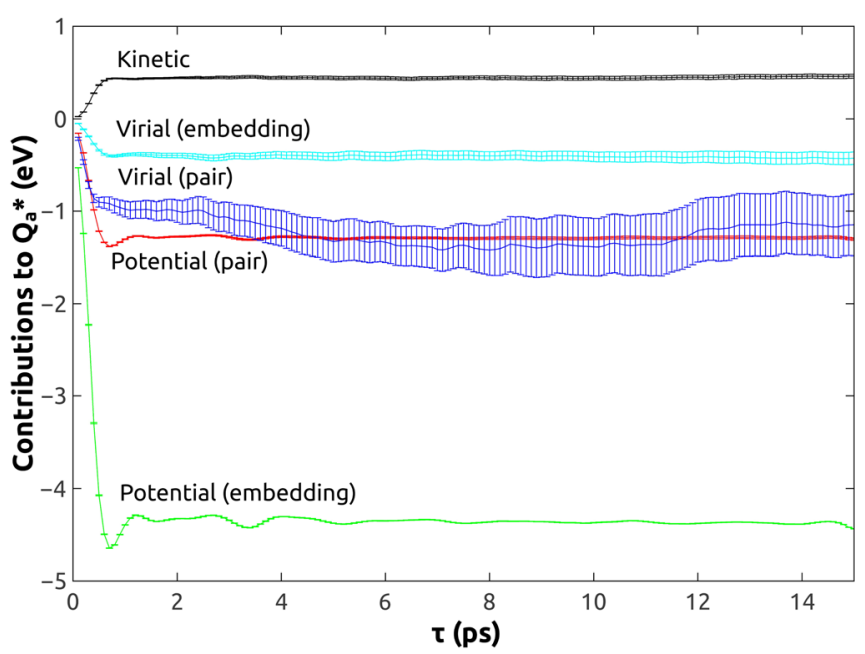

FIG. 5. Contributions to the integral in Eq. (2) as a function of integration limits $\tau$. Results correspond to the EAM potential for Au, with $T=700 \mathrm{~K}$. Three separate contributions to $q(t)$ due to the convective heat flux, including the kinetic energy (black line), pair potential (red line), and embedding energy (green line) are shown. The virial heat flux contributes to $q(t)$ due to the pair potential (dark blue line) and embedding energy (light blue line). Error bars shown are determined from statistical analysis of subsets of the ensemble.

direction as the atom hop. Rather, the heat flux becomes essentially zero in a time less than $\sim 1 \mathrm{ps}$ before and after the atom hop itself. This is to be compared to results obtained with pair potentials, where up to $\sim 10 \mathrm{ps}$ might be required for the heat flux to dissipate.

For pair and EAM potentials, comparison with reported experimental results for $Q_{a}^{* \prime}$ in Au is quite poor. The reported experimental values of $Q_{a}^{* \prime}$ for Au include $0.59 \mathrm{eV}$ from Ref. $15,0.68 \mathrm{eV}$ from Ref. 16, and $0.80 \mathrm{eV}$ from Ref. 17. The results for the Cherns potential, both here and from Ref. 9,

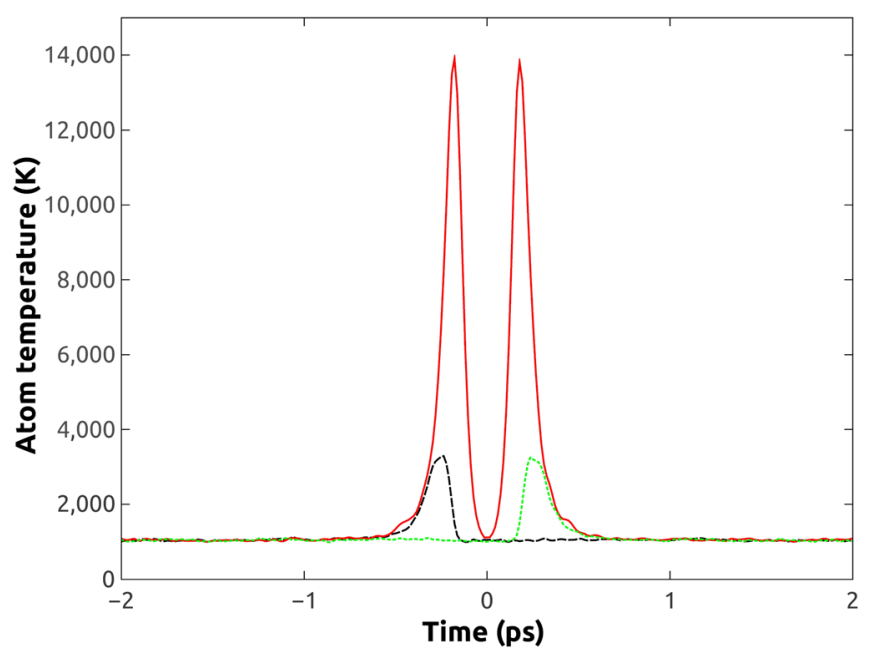

FIG. 6. Kinetic energy of the hopping atom (red curve) and two neighboring atoms along [110] plotted as an effective temperature. During the hop, the three atoms have kinetic energies significantly excited above the background temperature, computed here for the Morse potential with $\alpha r_{0}$ and average temperature $T=1016 \mathrm{~K}$. Before the hop $(t<0)$, the neighboring atom along [110] (black dashed line) is strongly excited, with its energy transferred to the hopping atom. After the hop $(t>0)$, energy is transferred from the hopping atom to the lattice, and the neighboring atom along [110] (green dotted line) becomes strongly excited.

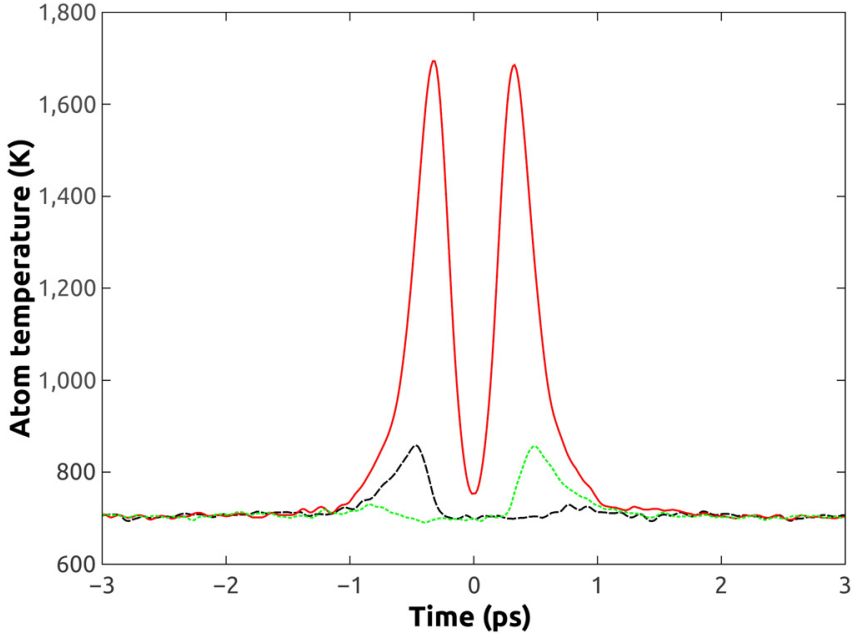

FIG. 7. Kinetic energy of the hopping atom (red curve) and two neighboring atoms along [110] plotted as an effective temperature. During the hop, the three atoms have kinetic energies significantly excited above the background temperature, computed here for the EAM potential for Au with average temperature $T=700 \mathrm{~K}$. Before the hop $(t<0)$, the neighboring atom along [110] (black dashed line) is strongly excited, with its energy transferred to the hopping atom. After the hop $(t>0)$, energy is transferred from the hopping atom to the lattice, and the neighboring atom along [110] (green dotted line) becomes strongly excited.

are significantly larger than experimental values. Similarly, the Morse potential with $\alpha r_{0}=4.55$ yields a value for $Q_{a}^{* \prime}$ that is far too large. While the pair potentials at least both yield positive values for $Q_{a}^{* \prime}$, the EAM potential gives a negative value with a fairly large magnitude.

Some insights into the hopping process and the role of the virial heat flux can be obtained by computing the kinetic energy of the hopping atoms and some of its neighbors. Specifically, we compute the kinetic energy of the hopping atom, averaged over the ensemble of successful hops, as well as the nearest neighbors along the [110] direction both before and after the hop. These are plotted in Fig. 6 for the Morse potential with $\alpha r_{0}=8.0$ at $T=1016 \mathrm{~K}$, and in Fig. 7 for the EAM potential with $T=700 \mathrm{~K}$. The kinetic energy of the atoms is represented as an effective temperature to facilitate comparison with the background temperature. In both cases, the hopping atom attains a very high kinetic energy. The maximum kinetic energy of the hopping atom is lower for the EAM potential, primarily due to the significantly lower activation energy $\left(U_{a c t}=0.60 \mathrm{eV}\right.$ for the EAM potential compared to $U_{a c t}=4.23 \mathrm{eV}$ for the Morse potential with $\left.\alpha r_{0}=8.0\right)$. However, there is evidence also that the friction force in the case of the EAM potentials is larger, thereby distributing the energy more efficiently to the lattice. In addition, in both Figs. 6 and 7, it is evident that the neighboring atoms are excited strongly. Specifically, the hopping atom receives energy mainly due to the large kinetic energy associated with its nearest neighbor along [110] before the hop. Moreover, the hopping atom loses a large amount of its kinetic energy to the nearest neighbor along [110] after the hop. In this way, the velocities of the hopping atom and its neighbors are very strongly correlated.

The general picture is that $Q_{a}^{* \prime}$ predicted using the Grout-Gillan method displays very strong dependence on the 
empirical potential and notable disagreement with experiment. Specifically, pair potentials tend to predict rather large positive values for $Q_{a}^{* \prime}$, whereas the EAM potential results in fairly large negative values for $Q_{a}^{* \prime}$, with both in disagreement with experiment. This suggests that many of the results previously reported using the Grout-Gillan method are particular features of pair potentials, and hence should be treated with caution. The EAM potential for Au, generally thought to more accurately simulate vacancies and diffusion in metals, results in qualitatively different behavior. However, agreement with experiment is not improved for the EAM potential. These observations suggest that there may be more fundamental issues associated with the GroutGillan method. This point is discussed in Sec. IV.

\section{ANALYSIS}

The results presented above demonstrate that pair and EAM potentials result in very different predictions for the computed value of $Q_{a}^{*}$ using the Grout-Gillan method. However, both cases result in strong disagreement with $Q_{a}^{* \prime}$ obtained in experiment. We believe that this is indicative of a fundamental issue with the Grout-Gillan method. As described here, the problem stems from the fact that the Grout-Gillan method samples only a limited ensemble of states with rather special properties, rather than an equilibrium ensemble which was implicit in the derivation of the method.

The relation $\vec{J}_{q}=Q_{a}^{*} \vec{J}_{a}$, which serves as the basis for the Grout-Gillan method, is derived under the assumption of an equilibrium statistical ensemble, with a nonzero $\vec{J}_{a}$ arising only due to concentration gradients and not temperature gradients. ${ }^{1}$ It is clear that the Grout-Gillan method, by only sampling states that result in an atomic hop, does not generate an equilibrium ensemble. Therefore, it needs to be proven that the ensemble generated by the Grout-Gillan method at least fulfills some general requirements, or equivalently that the neglected states are not required to compute the heat of transport using Eq. (1) and Eq. (2). Previous authors who have reported results obtained from the Grout-Gillan method have not addressed this point, and therefore in our view the method remains unproven.

Here, we highlight two basic properties of the equilibrium ensemble that are relevant in this context. Imagine many snapshots (i.e., an ensemble) of an equilibrium system containing many atoms which do not diffuse, and a single atom which represents the diffusing species. We assume here that atom $i=1$ labels the diffusing atom. The system can be taken to be in equilibrium with a reservoir at temperature $T$. First, it is a general requirement that the velocities $\vec{v}_{i}$ are uncorrelated with the atomic coordinates $\vec{r}_{i}$. The second general requirement is that the velocity components $v_{i \mu}$, where $\mu$ represents the Cartesian components of $\vec{v}_{i}$, be uncorrelated in the ensemble. Assuming a Boltzmann distribution of velocities, in equilibrium we require that

$$
\left\langle v_{i \mu} v_{j \nu}\right\rangle=\delta_{i, j} \delta_{\mu, \nu} \frac{k_{B} T}{m} .
$$

Next, we take for the microscopic definition of the mass flux of the diffusing atom $i=1$,

$$
\vec{J}_{1}=\vec{v}_{1}
$$

We consider the correlation between the energy flux $\vec{J}_{q}$ and mass flux $\vec{J}_{1}$ at the same time in the equilibrium ensemble. Based on the ensemble property in Eq. (27) and the microscopic definition of the energy flux, either Eq. (8) or Eq. (12), it is easy to show from that

$$
\left\langle\vec{J}_{q}(0) \cdot \vec{J}_{1}(0)\right\rangle=h_{1}\left\langle\vec{J}_{1}(0) \cdot \vec{J}_{1}(0)\right\rangle=\frac{3 h_{1} k_{B} T}{m},
$$

where $h_{1}$ is the partial enthalpy of the diffusing atom $i=1$. We have tested this prediction using the simulation model reported in Refs. 10 and 18 for hydrogen diffusing in a $\mathrm{Pd}$ lattice and found it to be exact. This is a distinct feature of diffusion in an equilibrium ensemble. It provides important insight into thermodiffusion, including the reason why experiments find that the heat of transport $Q_{a}^{*}$ tends to be not too dissimilar from the partial enthalpy of the diffusing species. This point will be revisited in the final section.

The ensemble which results from the Grout-Gillan method does not fulfill these two properties of the equilibrium ensemble. In the Grout-Gillan method, after the atom is released from the transition state, the velocity of the hopping atom and the surrounding atoms become strongly correlated, both in magnitude and direction. This fact is evident from the data plotted in Figs. 6 and 7. Specifically, in addition to a strong excitation of the hopping atom, the neighboring atoms along [110] are strongly excited during the atom hop. Moreover, the atom velocities are strongly correlated with the atom coordinates. The virial heat wave seen with GroutGillan, especially evident for pair potentials, is in fact due to strong velocity correlations in the ensemble.

To explain exactly why the Grout-Gillan ensemble does not yield correct predictions for the heat of transport, we can imagine a snapshot taken from the ensemble corresponding to the hopping atom with reaction coordinate $z$. In general, the velocity of the hopping atom will be strongly correlated to the velocities of the lattice atoms. However, we can imagine another state, equally likely to be observed in the equilibrium ensemble, but systematically neglected in the ensemble obtained using the Grout-Gillan method, in which we reverse the velocity vectors of only the lattice atoms, without changing their magnitude. While this additional state may not result in a successful hop, it will make a significant contribution to the energy flux, specifically tending to cancel out large contributions due to the virial energy flux. There is no justification for the neglect of this state, or any others, from the ensemble used to evaluate Eqs. (1) and (2).

What has been neglected in the Grout-Gillan method are states that are not related to an atomic hop, but nevertheless make contributions to the energy flux $\vec{J}_{q}$. Hence, Eqs. (1) and (2) are evaluated using the ensemble of states that does not reproduce the key features of the equilibrium ensemble outlined above, and hence $Q_{a}^{*}$ obtained from Eq. (2) cannot be related to the experimental heat of transport. At the present, it is unclear whether the Grout-Gillan method could be modified to result in correct predictions for $Q_{a}^{*}$. In our opinion, this is a difficult challenge, and alternatives to GroutGillan should be developed. 
The analytical result in Eq. (29) is closely related to calculation of $Q_{a}^{*}$ using the Green-Kubo approach ${ }^{20,21}$ and provides important insight into the problem. Specifically, Eq. (29) is suggestive as to why $Q_{a}^{*}$ is generally not dramatically different than the partial enthalpy of the diffusing species. Recently, we have studied thermodiffusion of hydrogen in Pd with analysis using the Green-Kubo method. ${ }^{10,18}$ These results have provided even more insight, albeit using pair potentials which are likely not predictive for the real system. Specifically, in Ref. 18, it was found that the correlations between energy and mass fluxes vanish in less than $\sim 0.5 \mathrm{ps}$. This suggests that any heat wave in the lattice does not play a dominant role. We attribute this to the fact that the GreenKubo method explores an equilibrium ensemble of states, unlike the special trajectories explored using Grout-Gillan. What was found in Ref. 18 was that the heat of transport can be interpreted as an effective partial enthalpy for those atoms of the hopping species with enough energy to overcome the activation barrier. By analyzing time-correlation functions of the individual contributions, it was established that the hopping hydrogen atom obtains an excess energy comparable to the migration barrier height. However, the virial term for a hopping atom, related to the stress component along the direction of the hop, was greatly reduced from that of an average hydrogen atom. In short, the results in Ref. 18 explain how various contributions tend to cancel, and consequently how the reduced heat of transport for interstitials might be either positive or negative, in general agreement with experiment. It is very likely that these results are not specific to hydrogen thermodiffusion, but rather are more general for both interstitial and vacancy-mediated thermodiffusion.

The picture that has emerged from this paper and Refs. 10 and 18 is that the reduced heat of transport $Q_{a}^{* \prime}$ generally reflects the excess partial enthalpy of diffusing atoms during a successful atomic hop. Lattice heat conduction likely plays a minor role, contrary to the predictions obtained using the Grout-Gillan method. The partial enthalpy of the diffusing species is usually thought of as a quantity averaged over the entire ensemble. In the context of thermodiffusion, it is best to consider a distribution of instantaneous partial enthalpies of the diffusing species. For atoms with an instantaneous enthalpy significantly different from the average partial enthalpy, successful hops become more likely. Hence, the heat of transport $Q_{a}^{*}$ represents the instantaneous partial enthalpy of diffusing atoms that are participating in a hop. Due to cancellations between convective and virial contributions, hopping atoms in an excited state and likely to hop may tend to have an instantaneous partial enthalpy either greater or less than the average value. This picture explains why the standard view that the reduced heat of transport is comparable to the activation energy $\Delta U_{\text {act }}$ has been found to be in disagreement with experiment, and also why the reduced heat of transport might be positive or negative.

Another relevant aspect of this problem is the role of electrons, which has hitherto been almost completely neglected. In metals, heat conduction is dominated by electrons, and the results obtained with the Grout-Gillan method suggest that thermodiffusion is intimately linked with ordinary heat conduction. Since the lattice heat wave should also be accompanied by heat conduction due to electrons, this makes the results obtained using pair potentials even more unlikely to be correct. Electronic heat conduction and lattice conduction together would seem to result in even larger heat conduction, and hence more positive values of $Q_{a}^{* \prime}$ that are in even stronger disagreement with experiment.

\section{CONCLUSIONS}

Two important results have emerged from this paper. These call into question previous applications of the GroutGillan method, including the use of pair potentials, and interpretation of the computed value $Q_{a}^{*}$ from Eqs. (1) and (2) as the heat of transport. This paper presents solid evidence that existing computational methods to determine the heat of transport for vacancy-mediated thermodiffusion have not been sufficiently established, and that further efforts are needed in this area. At present, there is no clear pathway to amending the basic approach of the Grout-Gillan method to correctly compute the heat of transport.

The first important result is that the large positive values found for $Q_{a}^{* \prime}$ obtained using the Grout-Gillan method here and in previous studies appear to be a distinct feature of pair potentials. When an EAM potential is used, there is no evidence of a heat wave occurring in the same direction as the atom hop. This particular behavior of the EAM potential appears to be related to the redistribution of embedding energy that occurs during the hop. Specifically, the change of atomic coordination which arises due to the atom hop results in a flow of energy in the opposite direction of the hop. Similar observations were made in a previous study of thermodiffusion of carbon interstitials in iron using an EAM potential and a method similar to Grout-Gillan. ${ }^{19}$ However, the EAM results presented here remain in very strong disagreement with experiment.

The second key result of this paper is the argument put forward which indicates that the quantity $Q_{a}^{*}$ computed in Eqs. (1)-(2) in the Grout-Gillan method should not be associated with the experimental heat of transport. Specifically, we have shown that the ensemble generated by the GroutGillan method does not obey key features required of an equilibrium statistical ensemble. The problem essentially arises from the neglect of states that, while not necessarily resulting in an atomic hop, nevertheless make significant contributions to the heat flux. In short, the basic premise in the Grout-Gillan method of only exploring successful hops has been demonstrated to be incorrect.

In attempting to find a way forward towards solving this problem, we finally comment on an alternative to GroutGillan, namely the "constrained-dynamics method," presented in two previous articles, ${ }^{10,22}$ which may be a way forward towards computing the heat of transport. This approach has resulted in predictions in closer agreement with experiment for vacancy thermodiffusion, ${ }^{22}$ and in the case of Pd:H essentially exact agreement with Green-Kubo methods. ${ }^{10}$ In this approach, the hopping species is dragged through the transition state in the presence of an applied temperature gradient using a constraint force. By computing the work done by the constraint force, it was shown in Ref. 22 that the heat of transport can be determined. By slowly dragging the hopping species through the transition state, the simulation 
samples an ensemble of states consistent with local equilibrium, and hence avoids the error of only sampling successful hopping trajectories made by the Grout-Gillan method. In particular, the constrained-dynamics method samples many states along the transition path that will not immediately yield successful atomic hops, and hence do not involve large correlations that should not be present in local equilibrium ensemble. This method we expect to be presented in more detail and with more applications in future articles.

\section{ACKNOWLEDGMENTS}

This work was supported by a grant from the National Science Foundation (Award No. 1106219). The authors acknowledge the University of Central Florida Stokes Advanced Research Computing Center for providing computational resources and support that have contributed to results reported herein. URL: http://webstokes.ist.ucf.edu

${ }^{1}$ R. E. Howard and A. B. Lidiard, "Matter transport in solids," Rep. Prog. Phys. 27, 161 (1964).

${ }^{2}$ M. J. Gillan, "The heat of transport in solids: A new theoretical approach," J. Phys. C: Sol. State Phys. 10, 1641 (1977).

${ }^{3}$ M. J. Gillan, "Heat of transport in solids: II. Harmonic theory for a vacancy in the simple cubic lattice," J. Phys. C: Sol. State Phys. 10, 3051 (1977).

${ }^{4}$ M. J. Gillan and M. W. Finnis, "Heat of transport in solids: III. Computer simulation of a vacancy in an fcc crystal," J. Phys. C: Sol. State Phys. 11, 4469 (1978).

${ }^{5}$ C. Jones, P. J. Grout, and A. B. Lidiard, "The heat of transport of vacancies in solid argon," Philos. Mag. Lett. 74, 217 (1996).

${ }^{6}$ C. Jones, P. J. Grout, and A. B. Lidiard, "The heat of transport of solute atoms in solid argon,” Ber. Busenges. Phys. Chem. 101, 1232 (1997).

${ }^{7}$ C. Jones, P. J. Grout, and A. B. Lidiard, "The heat of transport of vacancies in model fcc solids," Philos. Mag. A 79, 2051 (1999).
${ }^{8}$ P. J. Grout and A. B. Lidiard, "Computation of heats of transport in crystalline solids: II,” J. Phys. Condens. Matter 20, 425201 (2008).

${ }^{9}$ K. A. M. Dickens, P. J. Grout, and A. B. Lidiard, "Computation of heats of transport of vacancies in model crystalline solids: III," J. Phys. Cond. Matt. 23, 265401 (2011).

${ }^{10} \mathrm{P}$. K. Schelling and T. Le, "Computational methodology for analysis of the Soret effect in crystals: Application to hydrogen in palladium," J. Appl. Phys. 112, 083516 (2012).

${ }^{11}$ J. H. Irving and J. G. Kirkwood, "The statistical mechanical theory of transport processes. IV. The equations of hydrodynamics," J. Chem. Phys. 18, 817 (1950).

${ }^{12}$ D. Cherns, M. W. Finnis, and M. D. Matthews, "Sputtering of gold foils in a high voltage electron microscope: A comparison of theory and experiment," Philos. Mag. 35, 693 (1977).

${ }^{13} \mathrm{C}$. Kittel, Introduction to Solid State Physics, 8th edition, (John Wiley and Sons, Inc, Hoboken, NJ, 2005).

${ }^{14}$ G. Grochola, S. P. Russo, and I. K. Snook, "On fitting a gold embedded atom method potential using the force matching method," J. Chem. Phys. 123, 204719 (2005)

${ }^{15}$ D. Jaffe and P. G. Shewmon, Acta Metall. 12, 515 (1964).

${ }^{16} \mathrm{C}$. J. Meechan and G. W. Lehman, "Diffusion of $\mathrm{Au}$ and $\mathrm{Cu}$ in a temperature gradient," J. Appl. Phys. 33, 634 (1962).

${ }^{17}$ W. Mock, "Thermomigration of $\mathrm{Au}^{195}$ and $\mathrm{Sb}^{125}$ in Gold," Phys. Rev. 179, 663 (1969).

${ }^{18}$ W. C. Tucker, L. Shokeen, and P. K. Schelling, "Atomic-scale simulation of the thermodiffusion of hydrogen in palladium," J. Appl. Phys. 114, 063509 (2013).

${ }^{19}$ R. J. Asaro, D. Farkas, and Y. Kulkarni, "The Soret effect in diffusion in crystals," Acta Mat. 56, 1243 (2008).

${ }^{20}$ M. S. Green, "Markoff random processes and the statistical mechanics of time-dependent phenomena. 2. Irreversible processes in fluids," J. Chem. Phys. 22, 398 (1954).

${ }^{21}$ R. Kubo, "Statistical-mechanical theory of irreversible processes. 1. General theory and simple applications to magnetic and conduction problems," J. Phys. Soc. Japan 12, 570 (1957).

${ }^{22} \mathrm{Z}$. McDargh and P. K. Schelling, "Molecular-dynamics approach for determining the vacancy heat of transport," Comp. Mat. Sci. 50, 2363 (2011). 